Article

\title{
Chemical Analysis of the Sugar Moiety of Monohexosylceramide Contained in Koji, Japanese Traditional Rice Fermented with Aspergillus
}

\author{
Hiroshi Hamajima ${ }^{1, \dagger}{ }^{\dagger}$ Ayami Fujikawa ${ }^{1,+}$, Mikako Yamashiro ${ }^{1,+}$, Takatoshi Ogami ${ }^{2}$, \\ Seiichi Kitamura ${ }^{2}$, Masahito Tsubata ${ }^{2}$, Sei Tan ${ }^{1}$, Haruka Matsunaga ${ }^{1}$, Kazutaka Sawada ${ }^{3,4}$, \\ Satoshi Kumagai ${ }^{1}$, Nobuyuki Hayashi ${ }^{1}$, Koji Nagao ${ }^{1}$, Teruyoshi Yanagita ${ }^{5}$, Takuji Oka ${ }^{6}$, \\ Susumu Mitsutake ${ }^{1}$ and Hiroshi Kitagaki ${ }^{1,3, *}$ \\ Received: 13 August 2015; Accepted: 15 October 2015; Published: 2 January 2016 \\ Academic Editor: Badal C. Saha \\ 1 Faculty of Agriculture, Saga University, 1 Honjo-machi, Saga 840-8502, Japan; \\ hamajima@cc.saga-u.ac.jp (H.H.); 12252037@edu.cc.saga-u.ac.jp (A.F.); 11252059@edu.cc.saga-u.ac.jp (M.Y.); \\ 15555009@edu.cc.saga-u.ac.jp (S.T.); 15555013@edu.cc.saga-u.ac.jp (H.M.); kumagais@hotmail.co.jp (S.K.); \\ yurika@cc.saga-u.ac.jp (N.H.); knagao@cc.saga-u.ac.jp (K.N.); susumumi@cc.saga-u.ac.jp (S.M.) \\ 2 Research and Development Division, Toyo Shinyaku Co., Ltd., 7-28 Yayoigaoka, Tosu-shi, Saga 841-0005, \\ Japan; ogamit@toyoshinyaku.co.jp (T.O.); kitamuras@toyoshinyaku.co.jp (S.K.); \\ tsubatam@toyoshinyaku.co.jp (M.T.) \\ 3 Department of Biochemistry and Applied Biosciences, United Graduate School of Agricultural \\ Sciences Kagoshima, Kagoshima University, Korimoto 1-21-24, Kagoshima 890-8580, Japan; \\ sawada-kazutakaa@pref.saga.lg.jp \\ 4 Industrial Technology Center of Saga Prefecture, 114 Yaemizo, Saga 849-0932, Japan \\ 5 Faculty of Health and Nutrition Science, Nishikyushu University, 4490-9 Ozaki, Kanzaki, Saga 842-8585, \\ Japan; yanagitt@nisikyu-u.ac.jp \\ 6 Faculty of Biotechnology and Life Science, Sojo University, Ikeda 4-22-1, Kumamoto 860-0082, Japan; \\ oka@bio.sojo-u.ac.jp \\ * Correspondence: ktgkhrs@cc.saga-u.ac.jp; Tel.: +81-952-28-8766; Fax: +81-952-28-8709 \\ + These authors contributed equally to this work.
}

\begin{abstract}
Koji, rice fermented with Aspergillus, is used for saccharification of starch contained in crops during the manufacturing of many of Japanese traditional foods and drinks. Japanese people have long eaten koji, and many beneficial substances have been reported to be contained in koji. However, there has been no report on the existence or content of galactosylceramide in koji. To address this issue, we analyzed the chemical composition of the sugar moiety of monohexosylceramide contained in koji, and elucidate that $30.3 \%$ of yellow koji is galactosylceramide, $69.7 \%$ of that is glucosylceramide, $19.2 \%$ of white koji is galactosylceramide, and $80.8 \%$ of that is glucosylceramide. This is the first report of the existence and content of galactosylceramide in koji.
\end{abstract}

Keywords: koji; Aspergillus; glucosylceramide; galactosylceramide

\section{Introduction}

Koji is the traditional starch catalyst used for more than 1100 years in Japan. After rice crops are harvested, the surface of the crops is polished. The crops are steamed and the non-pathogenic fungus Aspergillus is inoculated to the crops. After fermenting for approximately $48 \mathrm{~h}$, the crops become koji. Koij is used for the manufacturing of many of Japanese traditional foods and drinks. The main islands of Japan use Aspergillus oryzae to manufacture koji, which is called yellow koji [1]. The southern islands, Kyushu and Okinawa, use Aspergillus luchuensis to manufacture koji, which is called white or black koji [2]. 
Koji is used for the manufacturing of many of Japanese traditional fermented foods. These include miso, soy sauce, sake, rice vinegar, black rice vinegar, salted koji, and mirin. Therefore, koji has a long eating history in Japan and is generally regarded as safe (GRAS) by the U.S. Food and Drug Administration. Koji is believed to have beneficial effects on health, regarding the longevity of the Japanese nation. Indeed, many beneficial substances such as ferrichrysin [3], $\beta$-glucan [4], dipeptidyl peptidase IV inhibitor [5], and kojic acid [6] have been reported to be contained in koji. However, the mechanism of the link between koji intake and health benefits has not been well described and the effect of health benefits of koji needs further research.

Sphingolipids are lipids which contain sphingoid bases and amide-linked fatty acids and contain various chemical forms [7,8]. Glycosylceramide is one form of sphingolipids. It contains a sugar moiety which is acetal-linked to the 1-hydrxyl position of the sphingoid base which constitutes ceramide. Fungi have various forms of glycosylceramide. Ascomycetes, including Aspergillus, Candida and Cryptococcus, have monoglycoside on the 1-hydroyl position of the sphingoid base [9-11]. On the contrary, zygomycetes such as Rhizopus have di- or tri-glycosides in this position [12]. Since koji and koji-derived foods contain $0.5-3 \mathrm{mg}$ of monohexosylceramide/g dry weight $[13,14]$, and Japanese people ingest approximately 28.4-66.0 mg monohexosylceramide per day, its health effects are of significant concern. Our research group and Fujino and Onishi have reported that the monohexosylceramide of Aspergillus is $N-2^{\prime}$-hydroxyoctadecanoyl-1-O- $\beta$-D-glucopyranosyl-9-methyl-4,8-sphingadienine $[9,10]$.

Many health benefits of sphingolipids have been reported. For example, the dietary intake of glucosylceramide prevents colon preneoplastic lesions [15] and head and neck cancers [16,17]. Also, when administered to the skin, it improves skin disorders [18]. Moreover, the dietary intake of sphingolipids also improves skin disorders [19-21].

Since galactosylceramide is rarely contained in foods, this might have a peculiar health benefit. However, there are very few studies on galactosylceramide, including the existence of galatosylceramide in koji or the functionality of galactosylceramide. Recently, it was reported that the mycelia of A. oryzae, A. sojae and A. awamori cultured in synthetic liquid media contain both $\beta$-glucosylceramide and $\beta$-galactosylceramide [11]. However, the growth and metabolism of Aspergillus in synthetic medium and that on rice are different [22]. Indeed, chemical compositions of the mycelia are reportedly different $[23,24]$. Therefore, it is necessary to analyze the actual content of the galactosylceramide contained in koji.

In this study, we tried to address the issue of which chemical forms of monohexosylceramides are contained in koji, the edible form of Aspergillus. It turned out that $30.3 \%$ of monohexosylceramide in yellow koji is galactosylceramide and $69.7 \%$ of that is glucosylceramide, and $19.2 \%$ of monohexosylceramide of white koji is galactosylceramide and $80.8 \%$ of that is glucosylceramide. This is the first report of the chemical composition of the sugar moiety of monohexosylceramide contained in koji, the edible form of Aspergillus.

\section{Material and Method}

\subsection{Materials}

The yellow koji and white koji were purchased from Tokushima seiko (Tokushima, Japan). Cerebroside (cb) was purchased from Matreya Inc. (Pleasant Gap, PA, USA). Glucose and galactose were purchased from Wako Pure Chemical Industries (Osaka, Japan).

\subsection{Thin Layer Chromatograph (TLC) Analysis}

Merck silicagel 60 plates (Merck Millipore, Darmstadt, Germany, Germany) were developed with the following solvents: chloroform:methanol:acetic acid:water (20:3.5:2.3:0.7, v/v) for developing monohexosylceramide, and chloroform:methanol:water (30:20:4, v/v) for developing simple sugars. Detection was performed with $2 \mathrm{mg} / \mathrm{mL}$ orcinol in $70 \% \mathrm{H}_{2} \mathrm{SO}_{4}$ reagent, and the plates were heated at $100{ }^{\circ} \mathrm{C}$ for $30 \mathrm{~min}$. 


\subsection{Extraction and Purification of Monohexosylceramide}

The total lipids were extracted from yellow koji and white koji using the method of modified Folch [25]. The total lipids were dried up and dissolved in hexane. Silicagel chromatograph was applied to the sample, after which it was eluted with ethyl acetate:methanol $(9: 1, \mathrm{v} / \mathrm{v})$ and fractionated by checking the elution positions of the monohexosylceramide using TLC. The fractions containing monohexosylceramide were dried up and dissolved in chloroform:methanol $(2: 1, \mathrm{v} / \mathrm{v})$. The sample was injected into the high-performance liquid chromatography (HPLC) system (LC-20AT, CTO-20AC, Shimadzu, Kyoto, Japan). The glycosylceranide purification by HPLC was performed using Inertsil SIL 100 A column $(5 \mu \mathrm{m}, 4.6 \mathrm{~mm}$ diameter $\times 250 \mathrm{~mm}$ length, GL science Inc., Tokyo, Japan). The mobile phase consisted of buffer A (chloroform) and buffer B ( $95 \%$ methanol), and separation was achieved using the following gradient program: $0 \mathrm{~min}$ A 100\%/B 0\%, $15 \mathrm{~min}$ A 75\%/B 25\%, and 20-40 min A $10 \% / B \quad 90 \%$. The flow rate was $0.7 \mathrm{~mL} / \mathrm{min}$, and the volume of the sample injection loop was $500 \mu \mathrm{L}$. Factions were collected at $1 \mathrm{~min}$ intervals and checked by TLC.

\subsection{Acid Hydrolysis of Monohexosylceramide}

The purified fraction containing monohexosylceramide was dried up and dissolved in $2 \mathrm{~N} \mathrm{H}_{2} \mathrm{SO}_{4}$, then it was incubated on heat block at $80{ }^{\circ} \mathrm{C}$ overnight. The hydrolysis product was added to chloroform and methanol and centrifuged at $1000 \mathrm{~g}$ for $10 \mathrm{~min}$. The upper water layer containing sugar was dried in freeze dryer and dissolved in water, and subjected to TLC analysis.

\subsection{High-Performance Liquid Chromatography (HPLC) Analysis}

The sugar detection by HPLC was performed using Aminex HPX-87H column $(7.8 \mathrm{~mm}$ diameter $\times 300 \mathrm{~mm}$ length, Biorad Laboratories, Hercules, CA, USA). The mobile phase consisted of $0.005 \mathrm{~mol} / \mathrm{L}$ sulfuric acid. The flow rate was $0.6 \mathrm{~mL} / \mathrm{min}$, and the volume of the sample injection was $20 \mu \mathrm{L}$. The detection of sugars was performed using refractive index (ERC Inc., Tokyo, Japan).

\section{Results and Discussion}

\subsection{Purification of Monohexosylceramide from Koji}

The extracted lipids from yellow koji and white koji were fractionated by silicagel chromatography. The fractions were collected at $10 \mathrm{~mL}$ intervals and the elution positions of the monohexosylceramide were checked using TLC (Figure 1). Monohexosylceramide, which we identified in previous studies [5,10], was eluted from fraction No. 7 to 19, but a monohexosylceramide fraction that does not contain other major contamination lipids appeared from fraction number 12. Therefore, the fractions No. 12-19 including monohexosylceramide were collected. The collected monohexosylceramide fractions were further purified with HPLC and checked with TLC (Figure 2). The amount of the purified yellow koji monohexosylceramide was approximately $900 \mu \mathrm{g}$, and that of white koji monohexosylceramide was approximately $1700 \mu \mathrm{g}$.

\subsection{Acid Hydrolysis of Monohexosylceramide}

The monohexosylceramides purified from yellow koji and white koji were hydrolyzed with $2 \mathrm{~N}$ sulfuric acid. The monohexosylceramide was degraded to sugar and ceramide (or sphingosine and fatty acid) by acid hydrolysis. The fractions were separated by phase break, and the upper layer, namely the aqueous phase, was collected and analyzed by TLC (Figure 3). The major portion of the sugar seemed to be glucose, and a small quantity of galactose was observed both in yellow koji and white koji. However, since the analysis was not quantitative, the amount of galactose was not known. Therefore, to quantitatively measure the amount of glucose and galactose, the fractions were analyzed by HPLC. 
$\mathbf{A}$
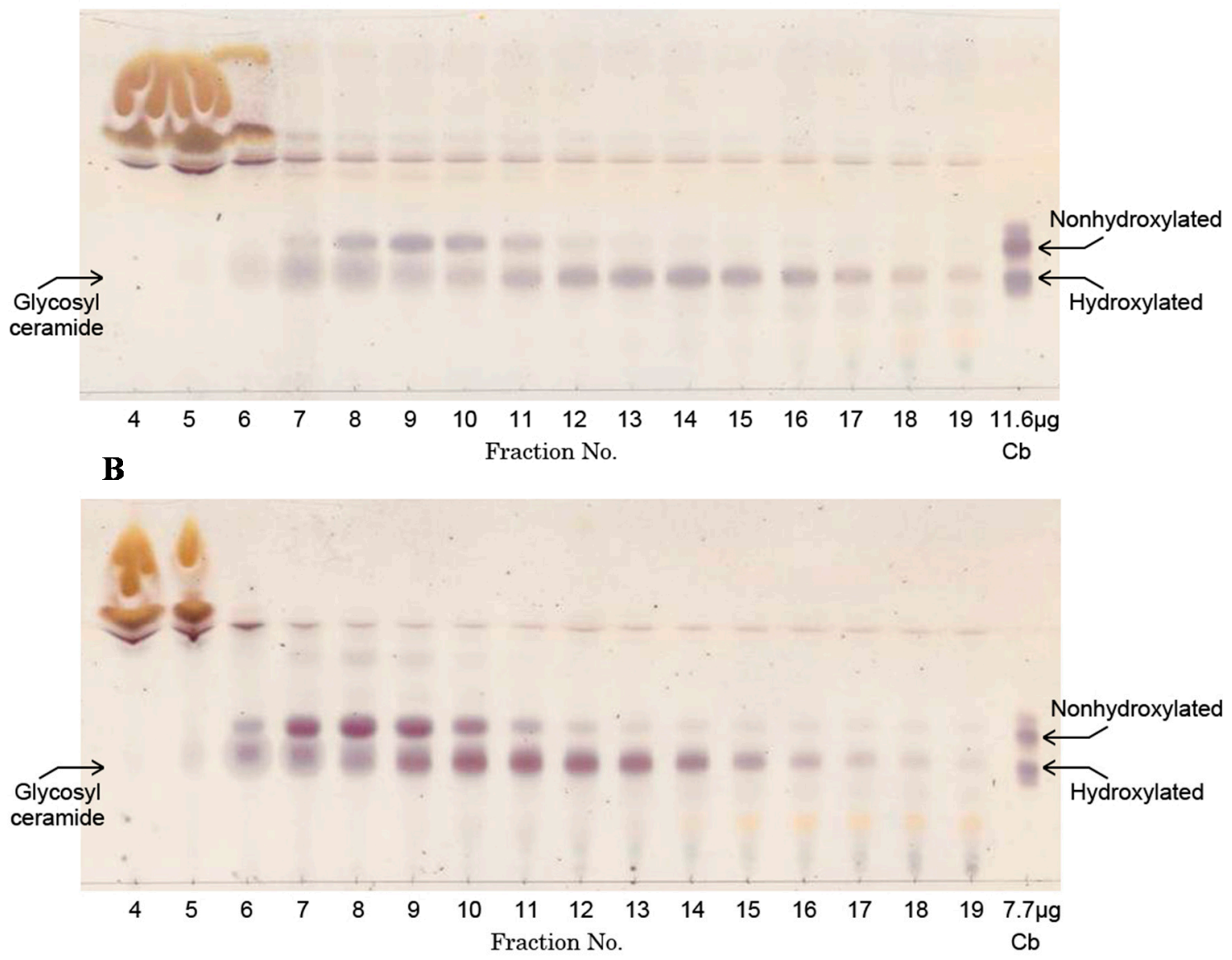

Figure 1. TLC analysis of lipids fractionated by silicagel chromatography. Lipids were extracted from yellow koji (A) and white koji (B) using chloroform/methanol (2:1 vol/vol), applied to silicagel, and eluted with ethyl acetate/methanol (9:1 vol/vol). The fractions were collected at $10 \mathrm{~mL}$ intervals and $100 \mu \mathrm{L}$ of the respective fractions were subjected to TLC analysis. $\mathrm{Cb}$ indicates standard cerebroside (Matreya Inc., Pleasant Gap, PA, USA).

$\mathbf{A}$

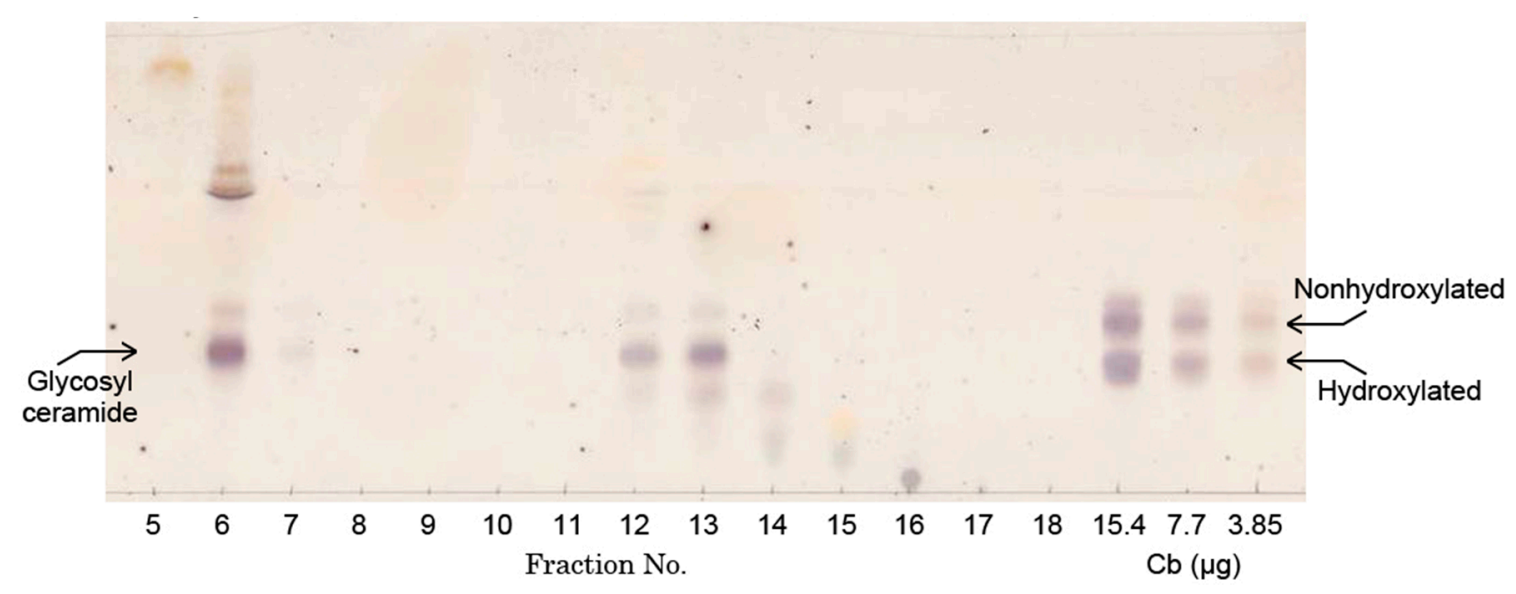

Figure 2. Cont. 
B

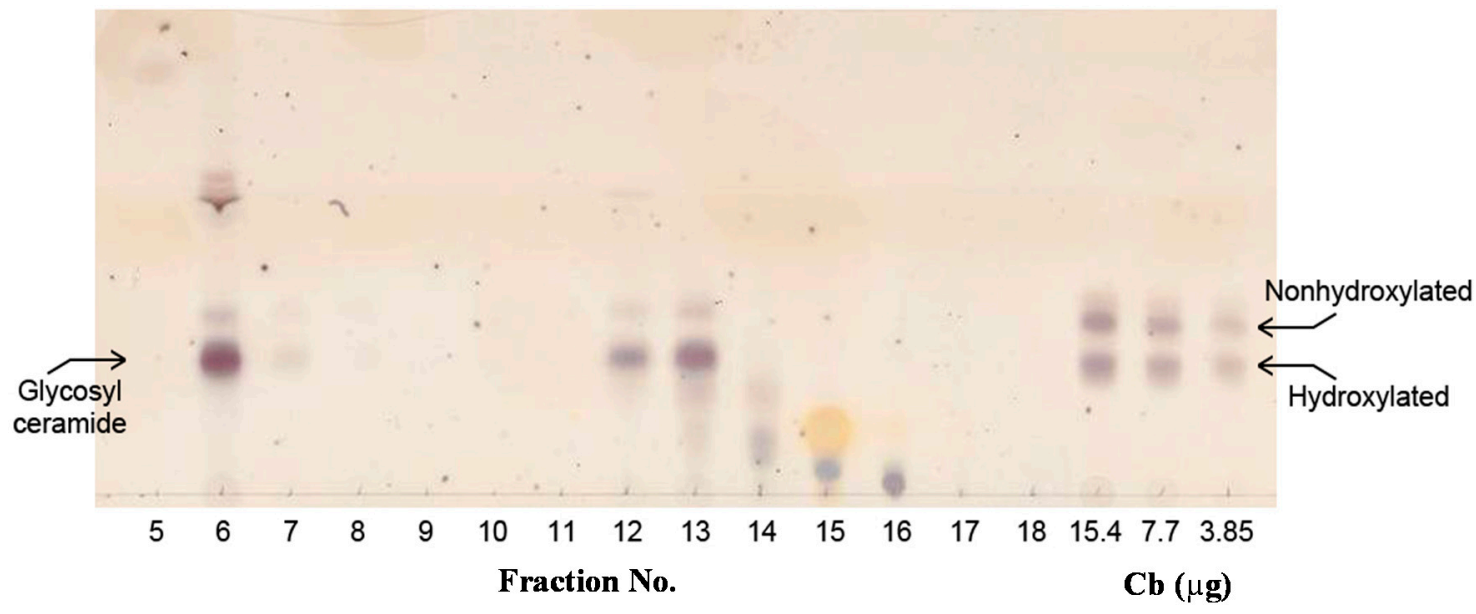

Figure 2. TLC analysis of purified monohexosylceramide by HPLC. Lipids were extracted from yellow koji (A) and white koji (B) and purified with HPLC (column: Inertsil $100 \mathrm{~A}$, elution: chloroform-95\% methanol, flow rate, $0.7 \mathrm{~mL} / \mathrm{min}$ ). The fractions were collected at $1 \mathrm{~min}$ intervals, and $100 \mu \mathrm{L}$ of the samples were subjected to TLC analysis. $\mathrm{Cb}$ indicates standard cerebroside (Matreya Inc., Pleasant Gap, PA, USA).

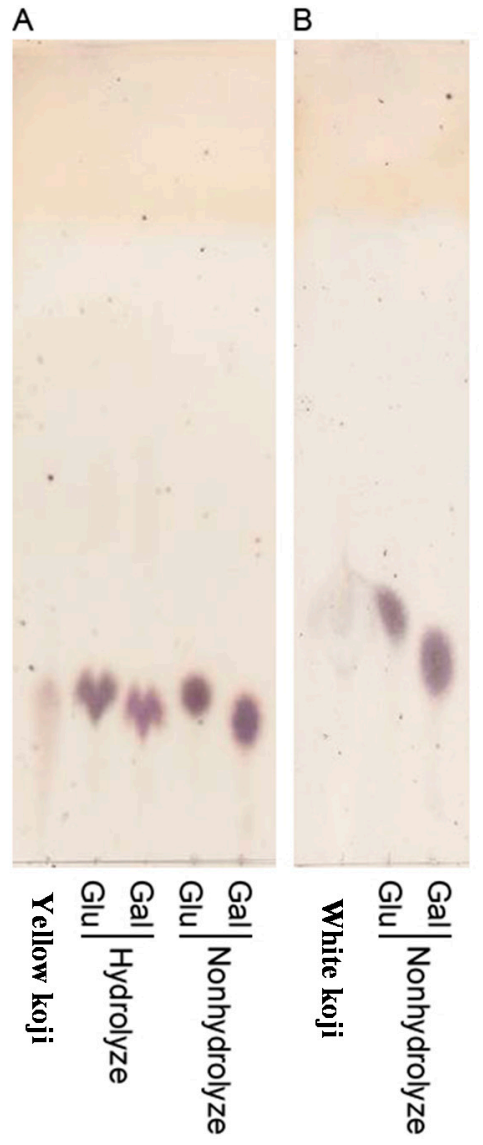

Figure 3. TLC analysis of the sugar moiety contained in monohexosylceramide purified from yellow and white koji. Monohexosylceramides purified from yellow (A) and white (B) koji, glucose (Glu) and galactose $(\mathrm{Gal})(10 \mu \mathrm{g})$ or those hydrolyzed with $2 \mathrm{~N}$ sulfuric acid at $80^{\circ} \mathrm{C}$ overnight were subjected to TLC analysis. 


\subsection{Quantitation of Sugars which Constitutes Koji Monohexosylceramide}

The above portions were analyzed by HPLC, which is equipped with a refractive index detector. As a result, both portions contained major glucose and minor galactose (Figure 4). Using the calibration curve, the sugar ratio of yellow koji was calculated to be glucose:galactose $=2.3: 1$ and that of white koji was calculated to be glucose:galactose $=4.2: 1$. Apparent degradation of glucose or galactose was not observed. These results indicate that $30.3 \%$ of the sugar moiety of monohexosylceramide of yellow koji is galactose, $69.7 \%$ of that is glucose, $19.2 \%$ of that of white koji is galactose and $80.8 \%$ of that is glucose (Figure 5). These results provide strong evidence that $70 \%-80 \%$ of monohexosylceramide of koji is $N$-2'-hydroxyoctadecanoyl-1-O- $\beta$-D-glucopyranosyl-9-methyl-4,8-sphingadienine and $20 \%-30 \%$ of that is $\mathrm{N}-2^{\prime}$-hydroxyoctadecanoyl-1-O- $\beta$-D-galactopyranosyl-9-methyl-4,8-sphingadienine.

A previous study by the other group [11] reported that $11.4 \mathrm{mg}$ of galactosylceramide and $10.6 \mathrm{mg}$ of glucosylceramide are contained in $30 \mathrm{~g}$ of mycelia of Aspergillus. The inconsistency of the ratio between their report and our report might be derived from the growth medium. The previous report adopted synthetic liquid medium, and the present report adopted rice crop as the growth medium of Aspergillus. Aspergillus might produce more galactosylceramide in liquid medium, which is a significant concern of the subsequent studies.

\section{A}
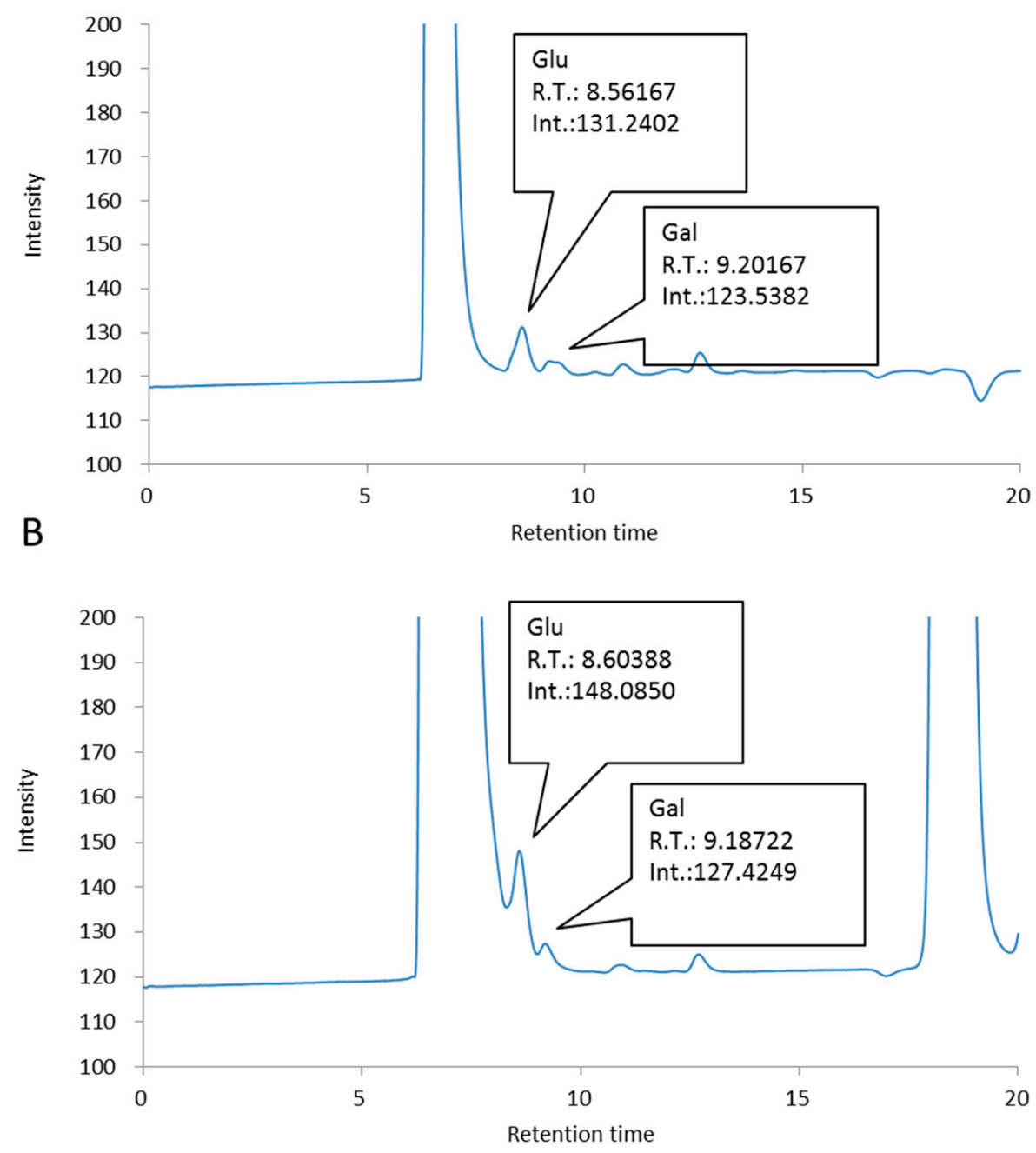

Figure 4. HPLC analysis of the sugar moiety contained in monohexosylceramide purified from yellow and white koji. (A) Yellow koji monohexosylceramide; (B) White koji monohexosylceramide. 
A

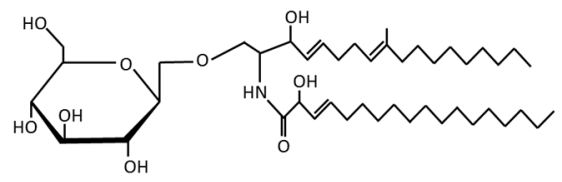

B

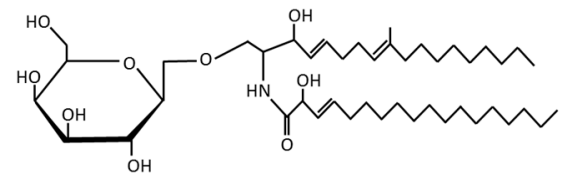

$\begin{array}{cc}\text { Yellow } & \text { White } \\ \text { koji } & \text { koji }\end{array}$

$69.7 \% \quad 80.8 \%$

$30.3 \% \quad 19.2 \%$

Figure 5. Compositions of the sugar moieties of monohexosylceramides contained in yellow and white koji. (A) Glucosylceramide; (B) Galactosylceramide.

\section{Conclusions}

The existence of the content of galactosylceramide in koji, the edible form of Aspergilus, was not reported until this study, although the Aspergillus mycelia contained a certain amount of galactosylceramide [11]. This study first elucidated that galactosylceramide is contained in koji at an amount of $19.2 \%-30.3 \%$ of total monohexosylceramide.

Considering the fact that Japanese people have long eaten 5-6 g of miso, which contains koji, per day [26], koji contains $0.5-3.0 \mathrm{mg}$ of monohexosylceramide per g dry weight, and the average Japanese meal contains $28.4-66.0 \mathrm{mg}$ of monohexosylceramide per day [27], the results elucidated in this study indicate that Japanese people have ingested $0.25-18 \mathrm{mg}$ of galactosylceramide per day for a long time. Although the health effects of galactosylceramide as food and/or cosmetics await further research, galactosylceramide might have a significant effect on the health of Japanese people.

Acknowledgments: This study was supported in part by the Scientific Technique Research Promotion Program for Agriculture, Forestry, Fisheries, and Food Industry (grant 26056A to H.K.) and by a JSPS KAKENHIgrant (grant 24580117, to H.K.). We would like to acknowledge Masatoshi Goto at Kyushu University for critical advices.

Author Contributions: Hiroshi Hamajima, Ayumi Fujikawa and Mikako Yamashiro performed degradation of monohexosylceramide. Takatoshi Ogami, Seiichi Kitamura and Masahito Tsubata performed cultures of Aspergillus. Sei Tan, Haruka Matsunaga and Kazutaka Sawada purified monohexosylceramide. Satoshi Kumagai and Nobuyuki Hayashi analyzed sugar content. Koji Nagao, Teruyoshi Yanagita, Takuji Oka and Susumu Mitsutake provided critical advice to the monohexosylceramide content of Aspergillus. Hiroshi Kitagaki supervised the research.

Conflicts of Interest: The authors declare no conflict of interest.

\section{References}

1. Kitagaki, H.; Kitamoto, K. Breeding researches of sake yeasts in Japan: History, recent technological advances, and future perspectives. Annu. Rev. Food. Sci. Technol. 2013, 4, 215-235. [CrossRef] [PubMed]

2. Hong, S.B.; Yamada, O.; Samson, R.A. Taxonomic re-evaluation of black koji molds. Appl. Microbiol. Biotechnol. 2014, 98, 555-561. [CrossRef] [PubMed]

3. Tadenuma, M.; Sato, S. Studies on the colorants in saké: Presence of ferrichrysin as iron containing colorant in sake. Agric. Biol. Chem. 1967, 31, 1482-1489. [CrossRef]

4. Das, A.; Raychaudhuri, U.; Chakraborty, R. Cereal based functional food of Indian subcontinent: A review. J. Food Sci. Technol. 2012, 49, 665-672. [CrossRef] [PubMed]

5. Imamura, K.; Tsuyama, Y.; Hirata, T.; Shiraishi, S.; Sakamoto, K.; Yamada, O.; Akita, O.; Shimoi, H. Identification of a gene involved in the synthesis of a dipeptidyl peptidase IV inhibitor in Aspergillus oryzae. Appl. Environ. Microbiol. 2012, 78, 6996-7002. [CrossRef] [PubMed]

6. Barnard, D.; Challenger, F. The formation of kojic acid (3-hydroxy-6-hydroxymethyl-gamma pyrone) from ethyl alcohol by Aspergillus oryzae. Biochem. J. 1946, 40, lxii. [PubMed]

7. Hannun, Y.A.; Obeid, L.M. Many ceramides. J. Biol. Chem. 2011, 286, 27855-27862. [CrossRef] [PubMed] 
8. Kitagaki, H.; Cowart, L.A.; Matmati, N.; vaena de Avalos, S.; Novgorodov, S.A.; Zeidan, Y.H.; Bielawski, J.; Obeid, L.M.; Hannun, Y.A. Isc1 regulates sphingolipid metabolism in yeast mitochondria. Biochim. Biophys. Acta 2007, 1768, 2849-2861. [CrossRef] [PubMed]

9. Hirata, M.; Tsuge, K.; Jayakody, L.N.; Urano, Y.; Sawada, K.; Inaba, S.; Nagao, K.; Kitagaki, H. Structural determination of glucosylceramides in the distillation remnants of shochu, the Japanese traditional liquor, and its production by Aspergillus kawachii. J. Agric. Food. Chem. 2012, 60, 11473-11482. [CrossRef] [PubMed]

10. Fujino, Y.; Ohnishi, M. Structure of cerebroside in Aspergillus oryzae. Biochim. Biophys. Acta 1976, 486, 161-171. [PubMed]

11. Tani, Y.; Amaishi, Y.; Funatsu, T.; Ito, M.; Itonori, S.; Hata, Y.; Ashida, H.; Yamamoto, K. Structural analysis of cerebrosides from Aspergillus fungi: The existence of galactosylceramide in A. oryzae. Biotechnol. Lett. 2014, 36, 2507-2513. [CrossRef] [PubMed]

12. Aoki, K.; Uchiyama, R.; Yamauchi, S.; Katayama, T.; Itonori, S.; Sugita, M.; Hada, N.; Yamada-Hada, J.; Takeda, T.; Kumagai, H.; et al. Newly discovered neutral glycosphingolipids in aureobasidin A-resistant zygomycetes: Identification of a novel family of Gala-series glycolipids with core Gal alpha 1-6Gal beta 1-6Gal beta sequences. J. Biol. Chem. 2004, 279, 32028-32034. [CrossRef] [PubMed]

13. Sawada, K.; Sato, T.; Hamajima, H.; Jayakody, L.N.; Hirata, M.; Yamashiro, M.; Tajima, M.; Mitsutake, S.; Nagao, K.; Tsuge, K.; et al. Glucosylceramide contained in Koji mold-cultured cereal confers membrane and flavor modification and stress tolerance to Saccharomyces cerevisiae during coculture fermentation. Appl. Environ. Microbiol. 2015, 81, 3688-3698. [CrossRef] [PubMed]

14. Takahashi, K.; Izumi, K.; Nakahata, E.; Hirata, M.; Sawada, K.; Tsuge, K.; Nagao, K.; Kitagaki, H. Quantitation and structural determination of glucosylceramides contained in sake lees. J. Oleo Sci. 2014, 63, 15-23. [CrossRef] [PubMed]

15. Inamine, M.; Suzui, M.; Morioka, T.; Kinjo, T.; Kaneshiro, T.; Sugishita, T.; Okada, T.; Yoshimi, N. Inhibitory effect of dietary monoglucosylceramide 1-O-beta-glucosyl- $\mathrm{N}$-2'-hydroxyarachidoyl-4,8-sphingadienine on two different categories of colon preneoplastic lesions induced by 1,2-dimethylhydrazine in F344 rats. Cancer Sci. 2005, 96, 876-881. [CrossRef] [PubMed]

16. Yazama, H.; Kitatani, K.; Fujiwara, K.; Kato, M.; Hashimoto-Nishimura, M.; Kawamoto, K.; Hasegawa, K.; Kitano, H.; Bielawska, A.; Bielawski, J.; et al. Dietary glucosylceramides suppress tumor growth in a mouse xenograft model of head and neck squamous cell carcinoma by the inhibition of angiogenesis through an increase in ceramide. Int. J. Clin. Oncol. 2015, 20, 438-446. [CrossRef] [PubMed]

17. Fujiwara, K.; Kitatani, K.; Fukushima, K.; Yazama, H.; Umehara, H.; Kikuchi, M.; Igarashi, Y.; Kitano, H.; Okazaki, T. Inhibitory effects of dietary glucosylceramides on squamous cell carcinoma of the head and neck in NOD/SCID mice. Int. J. Clin. Oncol. 2011, 16, 133-140. [CrossRef] [PubMed]

18. Kircik, L.; Hougeir, F.; Bikowski, J. Atopic dermatitis, and the role for a ceramide-dominant, physiologic lipid-based barrier repair emulsion. J. Drugs Dermatol. 2013, 12, 1024-1027. [PubMed]

19. Duan, J.; Sugawara, T.; Hirose, M.; Aida, K.; Sakai, S.; Fujii, A.; Hirata, T. Dietary sphingolipids improve skin barrier functions via the upregulation of ceramide synthases in the epidermis. Exp. Dermatol. 2012, 21, 448-452. [CrossRef] [PubMed]

20. Tsuji, K.; Mitsutake, S.; Ishikawa, J.; Takagi, Y.; Akiyama, M.; Shimizu, H.; Tomiyama, T.; Igarashi, Y. Dietary glucosylceramide improves skin barrier function in hairless mice. J. Dermatol. Sci. 2006, 44, 101-107. [CrossRef] [PubMed]

21. Takatori, R.; le Vu, P.; Iwamoto, T.; Satsu, H.; Totsuka, M.; Chida, K.; Shimizu, M. Effects of oral administration of glucosylceramide on gene expression changes in hairless mouse skin: Comparison of whole skin, epidermis, and dermis. Biosci. Biotechnol. Biochem. 2013, 77, 1882-1887. [CrossRef] [PubMed]

22. Yamane, Y.; Fujita, J.; Shimizu, R.; Hiyoshi, A.; Fukuda, H.; Kizaki, Y.; Wakabayashi, S. Production of cellulose- and xylan-degrading enzymes by a koji mold, Aspergillus oryzae, and their contribution to the maceration of rice endosperm cell wall. J. Biosci. Bioeng. 2002, 93, 9-14. [CrossRef]

23. Miranda, R.U.; Gómez-Quiroz, L.E.; Mendoza, M.; Pérez-Sánchez, A.; Fierro, F.; Barrios-González, J. Reactive oxygen species regulate lovastatin biosynthesis in Aspergillus terreus during submerged and solid-state fermentations. Fungal Biol. 2014, 118, 979-989. [CrossRef] [PubMed] 
24. Zhu, Y.X.; Yao, L.Y.; Jiao, R.H.; Lu, Y.H.; Tan, R.X. Enhanced production of Fumigaclavine C in liquid culture of Aspergillus fumigatus under a two-stage process. Bioresour Technol. 2014, 152, 162-168. [CrossRef] [PubMed]

25. Folch, J.; Lees, M.; Sloante Stanley, G.H. A simple method for the isolation and purification of total lipides from animal tissues. J. Biol. Chem. 1957, 226, 497-509. [PubMed]

26. Japan Federation of Miso Manufacturers' Cooperatives. Available online: http://www.zenmi.jp/ miso_toukei.html (accessed on 18 September 2015).

27. Yunoki, K.; Ogawa, T.; Ono, J.; Miyashita, R.; Aida, K.; Oda, Y.; Ohnishi, M. Analysis of sphingolipid classes and their contents in meals. Biosci Biotechnol Biochem. 2008, 72, 222-225. [CrossRef] [PubMed]

(C) 2016 by the authors; licensee MDPI, Basel, Switzerland. This article is an open access article distributed under the terms and conditions of the Creative Commons by Attribution (CC-BY) license (http://creativecommons.org/licenses/by/4.0/). 